\title{
Continuous Water-Quality and Suspended-Sediment Transport Monitoring in the San Francisco Bay, California, Water Years 2014-15
}

\author{
By Paul A. Buchanan, Maureen Downing-Kunz, David H. Schoellhamer, and Daniel N. Livsey
}

The U.S. Geological Survey (USGS) monitors water quality and suspended-sediment transport in the San Francisco Bay (bay) as part of a multi-agency effort to address management, water supply, and ecological concerns. The San Francisco Bay area is home to millions of people, and the bay teems both with resident and with migratory wildlife, plants, and fish. Freshwater mixes with salt water in the bay, which is subject both to riverine influences (floods, droughts, managed reservoir releases and freshwater diversions) and to marine influences (tides, waves, effects of salt water). To understand this environment, the USGS, along with its partners (see "Acknowledgements"), has been monitoring the bay's waters continuously since 1988. Several water-quality variables are of particular importance to State and Federal resource managers and are monitored at key locations throughout the bay (fig. 1). Salinity, which indicates the relative mixing of fresh and ocean waters in the bay, is derived from specific conductance measurements. Water temperature, along with salinity, affects the density of water, which controls gravity-driven circulation patterns and stratification in the water column. Turbidity, a measure of light scattered from suspended particles in the water, is used to estimate suspended-sediment concentration (SSC). Suspended sediment affects the bay in multiple ways: attenuation of sunlight in the water column, affecting phytoplankton growth; deposition on tidal marsh and intertidal mudflats, which can help sustain these habitats as sea level rises; deposition in ports and shipping channels, which can necessitate dredging; and often, adsorption of contaminants, affecting their distribution and concentrations in the environment. Dissolved oxygen concentration, essential to a healthy ecosystem and a fundamental indicator of water quality, is affected by water temperature, salinity, ecosystem metabolism, tidal currents, and wind. Tidal currents in the bay reverse four times a day, and wind direction and intensity typically vary on a daily cycle. Consequently, salinity, water temperature, SSC, and dissolved-oxygen concentration vary spatially and temporally throughout the bay. Therefore, continuous measurements are needed to observe these changes. The purpose of this fact sheet is to provide information about these variables, as well as internet links to access these continuous water-quality data collected by the USGS.

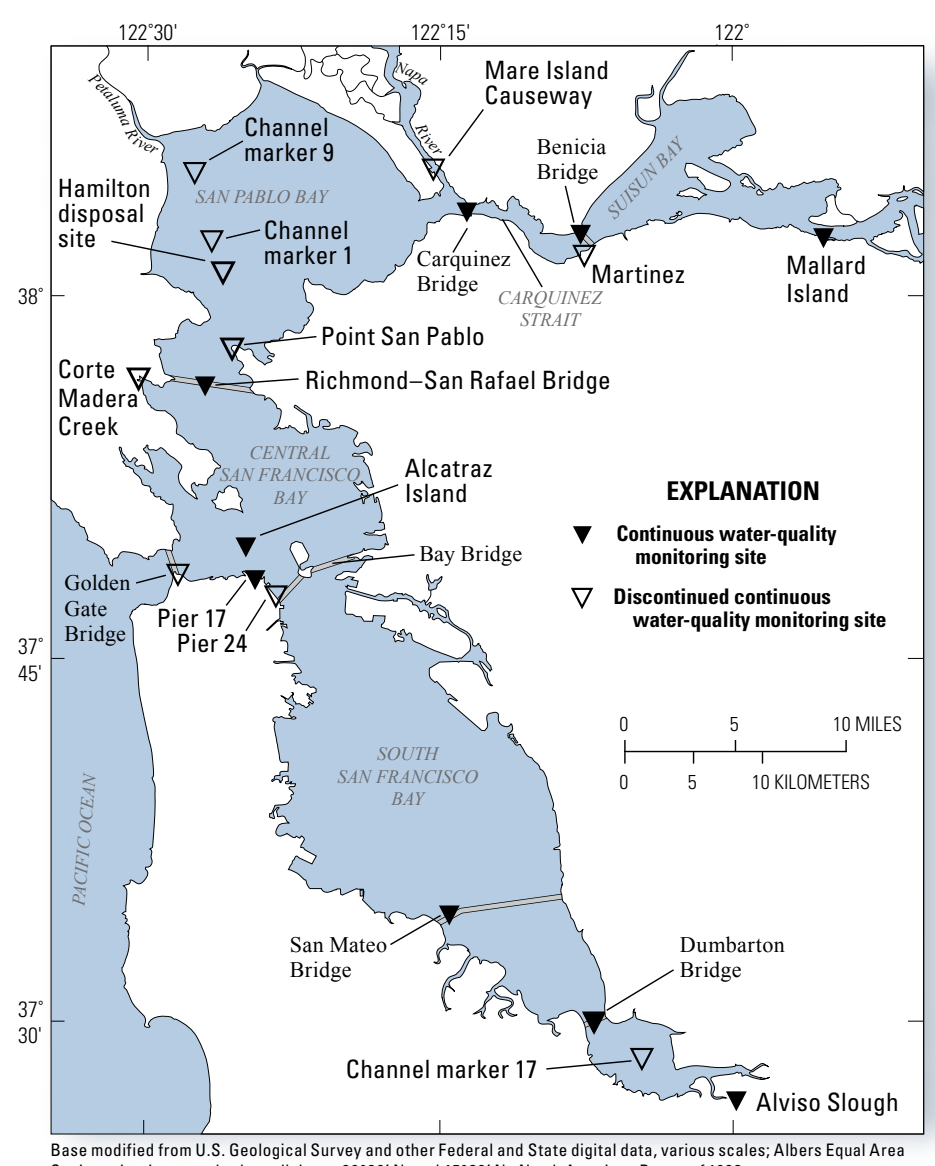

Base modified from U.S. Geological Survey and other Federal and State digital data, various scales
Conic projection, standard parallels are $29^{\circ} 30^{\circ}$ N. and $45^{\circ} 30^{\prime}$ N.; North American Datum of 1983.

Figure 1. San Francisco Bay study area, California.

\section{Program Overview}

Continuous water-quality measurements are, or have been, collected at several monitoring stations in the bay (fig. 1; table 1). Typically, instruments are suspended in the water from a stainless-steel cable that is anchored to the bottom (fig. 2) and are equipped with a variety of sensors. Data are recorded every 15 minutes and are retrieved either by cellular telemetry (yielding provisional data available within 1 hour of measurement) or during periodic site visits (yielding provisional data available within 1 week of the site visit). Biological growth, which can affect sensor readings, usually increases with time, and the affected data need to be revised or deleted. Every $2-5$ weeks, each site is visited to clean and calibrate the instruments and retrieve data as needed. Water samples 
are collected at the sensor depth to relate the turbidity data to SSC (fig. 3). For stations where water discharge is computed along with cross-sectionally averaged SSC, water discharge is measured by using a boat-mounted acoustic Doppler current profiler (Mueller and others, 2013), and water samples are collected periodically at points across the channel by the equal-discharge-increment method (fig. 4; U.S. Geological Survey, 2006). Suspended-sediment flux, in mass per unit time, is computed as the product of the water discharge and the

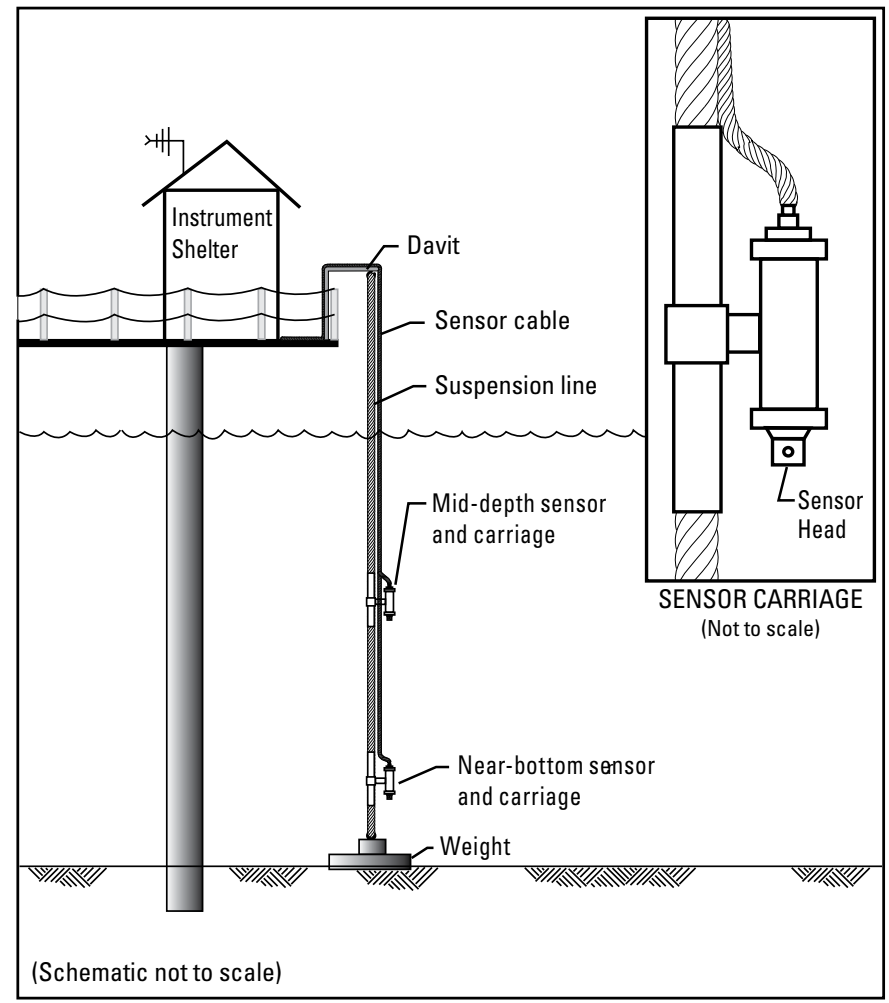

Figure 2. Typical monitoring installation, San Francisco Bay study.

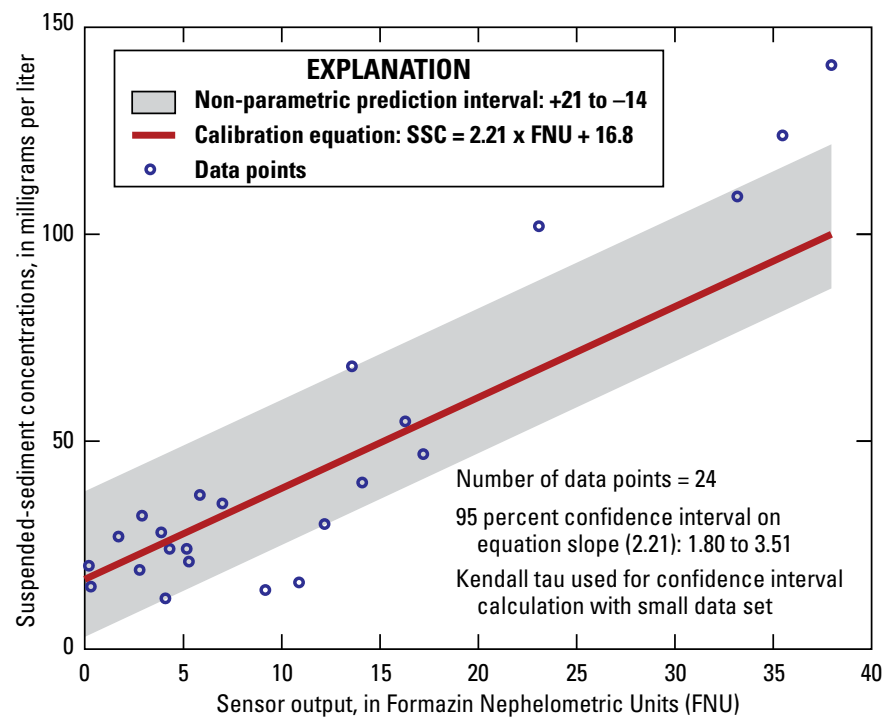

Figure 3. Example rating curve that relates turbidity to suspendedsediment concentrations (SSC) using the nonparametric repeated median method (Siegel, 1982). Output of an optical turbidity sensor is related to the SSC measured in water samples. channel-average SSC at the indicated cross section. Data are edited and reviewed before final approval. Further details about these methods are available at https:/ca.water.usgs.gov/projects/ baydelta (access the "Methods" section).

Specific-conductance, water-temperature and turbidity data are collected at two depths in the water column to help characterize the vertical variability. For stations in shallow water (Alviso Slough, Pier 17, and Alcatraz Island), data are collected only at one depth. Dissolved-oxygen data are only collected at Alviso Slough (table 1).

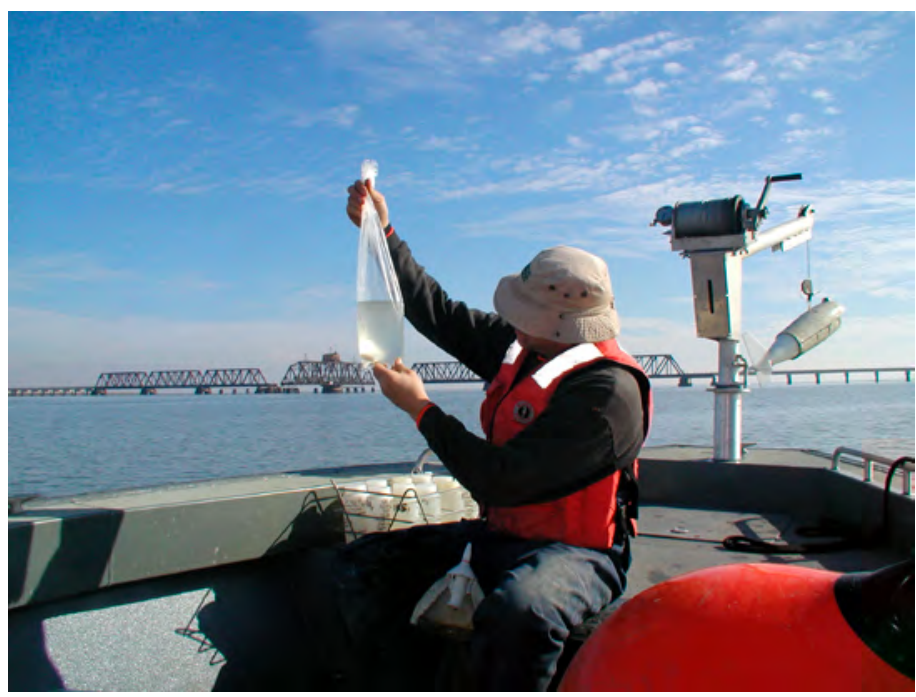

Figure 4. U.S. Geological Survey scientist collecting a suspendedsediment concentration sample with a depth-integrated sampler at South San Francisco Bay near the Dumbarton Bridge. Photograph taken by Greg Shellenbarger.

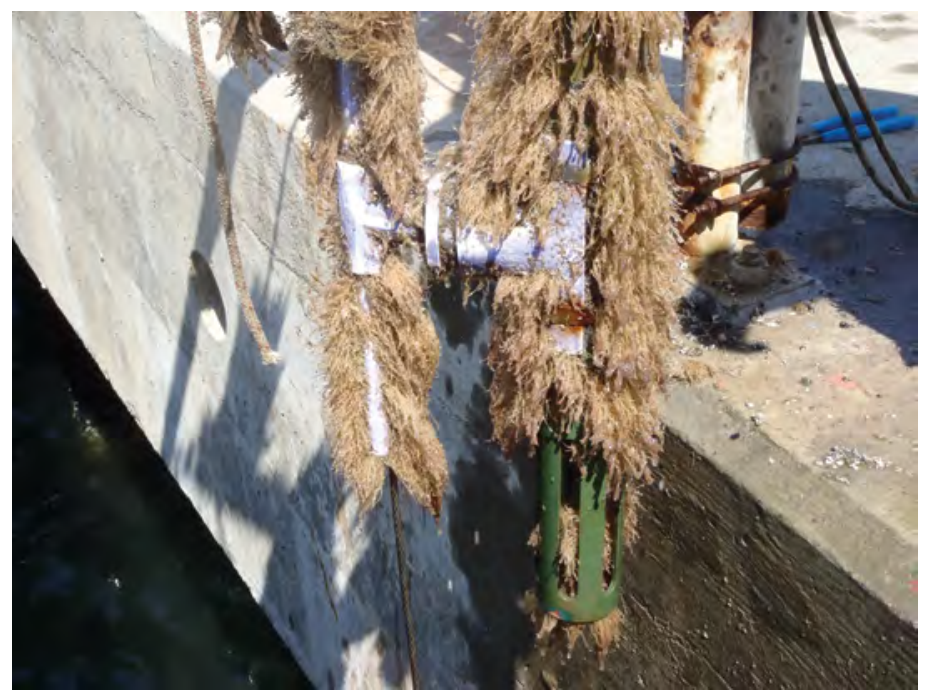

Figure 5. Biological fouling on water-quality instruments at the Richmond-San Rafael Bridge. 


\section{Instrument Specifications}

Specific conductance (reported in microsiemens per centimeter at 25 degrees Celsius) and water temperature (reported in degrees Celsius) have been measured using the YSI, Inc., 6560 conductance/temperature sensor. ${ }^{1}$ Two types of optical sensors have been used to measure turbidity: the DTS-12, manufactured by Forest Technology Systems, and model 6136, manufactured by YSI, Inc. Dissolved oxygen has been measured by the optical sensor model 6150 , manufactured by YSI, Inc. Sensors manufactured by YSI, Inc., are installed on the 6920 multi-parameter water-quality instrument.

In a continuous water-quality monitoring program, potential sources of error include, but are not limited to, electronic drift, calibration errors, and biological fouling of sensors (fig. 5). Data corrections (necessary because of biological fouling or instrument electronic drift) have been applied to the affected periods of record following USGS guidelines (https://ca.water. usgs.gov/projects/baydelta).

Continuous water-quality and suspended-sediment transport data collected during water years 2014 and 2015 (October 2013September 2015) are archived in the USGS National Water Information System and are available to the public at https://waterdata.usgs.gov/ca/nwis.

For additional information:

https://ca.water.usgs.gov/projects/baydelta/

Contact:

Paul Buchanan

California Water Science Center

U.S. Geological Survey

6000 J Street, Placer Hall, Sacramento, CA 95819

buchanan@usgs.gov

\section{Acknowledgements}

Collection of these data was supported by the U.S. Army Corps of Engineers, San Francisco District, as part of the Regional Monitoring and Regional Sediment Management Programs; Interagency Ecological Program; California State Coastal Conservancy; Bureau of Reclamation; U.S. Geological Survey Priority Landscapes Program; and the U.S. Geological Survey Federal/State Cooperative Program.

\section{References}

Mueller, D.S., Wagner, C.R., Rehmel, M.S., Oberg, K.A., and Rainville, F., 2013, Measuring discharge with acoustic Doppler current profilers from a moving boat (ver. 2.0, December 2013): U.S. Geological Survey Techniques and Methods, book 3, chap. A22, 95 p., https://dx.doi.org/10.3133/tm3A22.

Siegel, A.R., 1982, Robust regression using repeated medians: Biometrika, v. 69, p. 242-244.

U.S. Geological Survey, 2006, Collection of water samples (ver. 2.0): U.S. Geological Survey Techniques of WaterResources Investigations, book 9, chap. A4, p. 50-58, September 2006, accessed August 24, 2017, at http://pubs.water.usgs.gov/twri9A4/.
${ }^{1}$ The use of firm, trade, and brand names in this report is for identification purposes only and does not constitute endorsement by the U.S. Government. 
Table 1. Continuous water-quality monitoring stations, Suisun Bay, San Pablo Bay, and Central and South San Francisco Bays, California, 0ctober 1, $2013-$ September 30, 2015.

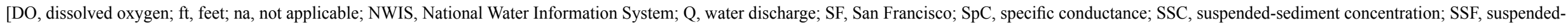
sediment flux; T, water temperature; $\mathrm{X}$, collected parameter; $\mu \mathrm{S} / \mathrm{cm}$, microsiemens per centimeter; ${ }^{\circ} \mathrm{C}$, degrees Celsius; - , no data]

\begin{tabular}{|c|c|c|c|c|c|c|c|c|c|}
\hline \multirow{2}{*}{$\begin{array}{l}\text { Measurement } \\
\text { location }\end{array}$} & & \multicolumn{6}{|c|}{ Water-quality parameter } & \multirow{2}{*}{$\begin{array}{l}\text { Start of } \\
\text { record } \\
\text { (water year) }\end{array}$} & \multirow[b]{2}{*}{ Remarks } \\
\hline & & $\begin{array}{c}\text { Specific } \\
\text { conductance }\end{array}$ & Temperature & Turbidity & SSC & $\begin{array}{l}0, \\
\text { SSF }\end{array}$ & $\begin{array}{c}\text { Dissolved } \\
\text { oxygen }\end{array}$ & & \\
\hline \multicolumn{10}{|c|}{ Alviso Slough near Alviso, 11169750} \\
\hline Near bottom & $\begin{array}{l}\text { Percent valid } \\
\text { data* }\end{array}$ & 82 & 89 & 78 & - & - & 88 & 2010 & $\begin{array}{l}\text { Highest SpC for period of record: September } 12 \text { and } 13,2015 \\
\quad(48,700 \mu \mathrm{S} / \mathrm{cm}) \text {. }\end{array}$ \\
\hline Cross-section & - & - & - & - & $\mathrm{X}$ & $\mathrm{X}$ & na & 2010 & Q and SSF are not yet available from NWIS. \\
\hline \multicolumn{10}{|c|}{ South SF Bay at Dumbarton Bridge, 373015122071000} \\
\hline Upper & $\begin{array}{l}\text { Percent valid } \\
\text { data* }^{\text {Pat }}\end{array}$ & - & - & 61 & 61 & - & na & 1993 & Data are downloaded hourly using cellular telemetry. \\
\hline Lower & $\begin{array}{l}\text { Percent valid } \\
\text { data* }\end{array}$ & - & - & 64 & 64 & - & na & 1993 & Dissolved-oxygen data collection was discontinued on October 1, 2014. \\
\hline Cross-section & - & - & - & - & - & $\mathrm{X}$ & na & 2009 & Q and SSF data are not yet available from NWIS. \\
\hline \multicolumn{10}{|c|}{ SF Bay at San Mateo Bridge near Foster City, 11162765} \\
\hline Upper & $\begin{array}{l}\text { Percent valid } \\
\text { data* }^{*}\end{array}$ & 86 & 94 & - & - & - & na & 1989 & $\begin{array}{l}\text { Data are downloaded hourly using cellular telemetry. } \\
\text { Highest SpC for period of record: September } 9,2014(51,100 \mu \mathrm{S} / \mathrm{cm}) \\
\text { Highest T for period of record: August } 7,2015\left(24.0^{\circ} \mathrm{C}\right)\end{array}$ \\
\hline Lower & $\begin{array}{l}\text { Percent valid } \\
\text { data* }\end{array}$ & 63 & 88 & - & - & - & na & 1989 & $\begin{array}{l}\text { Highest SpC for period of record: September } 20,21,23-26,2014(51,300 \mu \mathrm{S} / \mathrm{cm}) \text {. } \\
\text { Highest T for period of record: July } 27,2014\left(23.9^{\circ} \mathrm{C}\right) \text {. } \\
\text { Dissolved-oxygen data collection was discontinued October 1, } 2014 .\end{array}$ \\
\hline \multicolumn{10}{|c|}{ SF Bay at Pier 17,374811122235001} \\
\hline Near bottom & $\begin{array}{l}\text { Percent valid } \\
\text { data* }\end{array}$ & 79 & 85 & 81 & 81 & - & na & 2014 & $\begin{array}{l}\text { Highest SpC for period of record: July } 8,9,11,13,2014(49,900 \mu \mathrm{S} / \mathrm{cm}) \text {. } \\
\text { Highest T for period of record: August } 29,2015\left(20.4{ }^{\circ} \mathrm{C}\right) . \\
\text { Data are downloaded hourly using cellular telemetry. } \\
\text { Dissolved-oxygen data collection was discontinued October } 1,2014 .\end{array}$ \\
\hline \multicolumn{10}{|c|}{ SF Bay at Alcatraz Island, 374938122251801} \\
\hline Mid-depth & $\begin{array}{l}\text { Percent valid } \\
\text { data* }\end{array}$ & 85 & 98 & 72 & 72 & - & na & 2003 & $\begin{array}{l}\text { Data are downloaded hourly using cellular telemetry. } \\
\text { Highest SpC for period of record: August } 29,2008(49,300 \mu \mathrm{S} / \mathrm{cm}) . \\
\text { Highest } \mathrm{T} \text { for period of record: July } 26,2014 \text {, August } 28,2015\left(20.1^{\circ} \mathrm{C}\right) \text {. }\end{array}$ \\
\hline \multicolumn{10}{|c|}{ SF Bay at Richmond-San Rafael Bridge, 375607122264701} \\
\hline Upper & $\begin{array}{l}\text { Percent valid } \\
\text { data* }\end{array}$ & 87 & 87 & 84 & 84 & - & na & 2006 & $\begin{array}{l}\text { Highest SpC for period of record: June 14, } 2004(51,000 \mu \mathrm{S} / \mathrm{cm}) \text {. } \\
\text { Highest T for period of record: August 17, } 2015\left(21.9^{\circ} \mathrm{C}\right) .\end{array}$ \\
\hline
\end{tabular}


Table 1. Continuous water-quality monitoring stations, Suisun Bay, San Pablo Bay, and central and south San Francisco Bays, California, October 1, 2013-September 30, 2015.-Continued

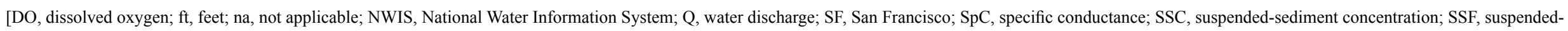
sediment flux; T, water temperature; $\mathrm{X}$, collected parameter; $\mu \mathrm{S} / \mathrm{cm}$, microsiemens per centimeter; ${ }^{\circ} \mathrm{C}$, degrees Celsius; - , no data]

\begin{tabular}{|c|c|c|c|c|c|c|c|c|c|}
\hline \multirow{2}{*}{$\begin{array}{l}\text { Measurement } \\
\text { location }\end{array}$} & & \multicolumn{6}{|c|}{ Water-quality parameter } & \multirow{2}{*}{$\begin{array}{c}\text { Start of } \\
\text { record } \\
\text { (water year) }\end{array}$} & \multirow[b]{2}{*}{ Remarks } \\
\hline & & $\begin{array}{c}\text { Specific } \\
\text { conductance }\end{array}$ & Temperature & Turbidity & SSC & $\begin{array}{c}0, \\
\text { SSF }\end{array}$ & $\begin{array}{c}\text { Dissolved } \\
\text { oxygen }\end{array}$ & & \\
\hline \multicolumn{10}{|c|}{ Carquinez Strait at Carquinez Bridge, 11455820} \\
\hline Upper & $\begin{array}{l}\text { Percent valid } \\
\text { data* }^{*}\end{array}$ & 64 & 66 & - & - & - & na & 1998 & $\begin{array}{l}\text { Highest } \mathrm{SpC} \text { for period of record: October 9, 2014, October 16, } 2015 \\
\quad(43,400 \mu \mathrm{S} / \mathrm{cm})\end{array}$ \\
\hline Lower & $\begin{array}{l}\text { Percent valid } \\
\text { data* }^{*}\end{array}$ & 68 & 69 & - & - & - & na & 1999 & Highest SpC for period of record: August 29, September 3, $2015(44,400 \mu \mathrm{S} / \mathrm{cm})$. \\
\hline \multicolumn{10}{|c|}{ Suisun Bay at Benicia Bridge, 11455780} \\
\hline Upper & $\begin{array}{l}\text { Percent valid } \\
\text { data* }^{*}\end{array}$ & 93 & 98 & 81 & 81 & - & na & 2001 & $\begin{array}{l}\text { Data are downloaded hourly using cellular telemetry. } \\
\text { Highest } \mathrm{SpC} \text { for period of record: July 31, } 2015(38,700 \mu \mathrm{S} / \mathrm{cm}) \text {. }\end{array}$ \\
\hline Lower & $\begin{array}{l}\text { Percent valid } \\
\text { data* }^{*}\end{array}$ & 92 & 96 & 85 & 85 & - & na & 2001 & $\begin{array}{l}\text { Highest SpC for period of record: October 14, } 2014(40,100 \mu \mathrm{S} / \mathrm{cm}) \text {. } \\
\text { Dissolved-oxygen data collection was discontinued October 1, } 2014 .\end{array}$ \\
\hline \multicolumn{10}{|c|}{ Suisun Bay at Mallard Island, 11185185} \\
\hline Upper & $\begin{array}{l}\text { Percent valid } \\
\text { data* }^{*}\end{array}$ & - & - & 95 & 95 & - & na & 1994 & $\begin{array}{l}\text { Data are downloaded hourly using cellular telemetry. } \\
\text { Upper sensor attached to a float to maintain a constant depth below water surface } \\
\text { of } 3.3 \mathrm{ft} \text {. }\end{array}$ \\
\hline Lower & $\begin{array}{l}\text { Percent valid } \\
\text { data* }^{*}\end{array}$ & - & - & 98 & 98 & - & na & 1994 & - \\
\hline
\end{tabular}

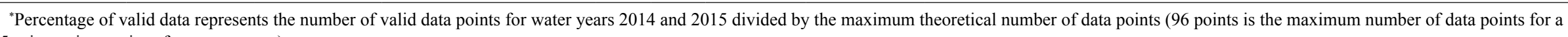
15 -minute time series of measurements). 